\title{
Working Memory Capacity and the Cognitive Underpinnings of Syntactic Processing
}

Polly O’Rourke (Center for the Advanced Study of Language, University of Maryland)

\section{Introduction}

Working memory (WM) is "a multicomponent system responsible for active maintenance of information in the face of ongoing processing and/or distraction” (Conway et al. 2005, p. 770) which facilitates goal directed behavior. It consists of numerous mechanisms including the ability to maintain/divide attention, monitor for information, maintain/update information and inhibit prepotent responses during task performance. WM capacity (WMC) predicts general fluid intelligence (Conway, Cownan, Bunting, Therriault \& Minkoff, 2002) and certain aspects language processing performance (Chiappe \& Chiappe, 2007; Just \& Carpenter, 1992; Daneman \& Carpenter, 1980; Caplan \& Waters, 1999). Many studies have shown that individuals with greater WMC show better comprehension performance of syntactically complex sentences (e.g. garden-path and object relative structures) than low WMC individuals (Friederici, Steinhauer, Mecklinger \& Meyer, 1998; Just \& Carpenter, 1992; King \& Just, 1991; Vos, Gunter, Schriefers \& Friederici, 2001).

Understanding of the relationship between WMC and different types of syntactic processing is limited by the fact that the vast majority of studies that have examined the connection have used one task, the reading span task (Danemann \& Carpenter, 1980), as the sole index of WMC while many different assessments, which tap different working memory mechanisms, exist (Conway, Kane, Bunting, Hambrick, Wilhelm \& Engle, 2005). The goal of the current study was to determine if working memory mechanisms other than those indexed by reading span are relevant to the comprehension of difficult syntax. To this end, WMC was assessed using four different measures: reading span, operation span, $n$-back and anti-saccade. The reading span task assesses the ability to maintain and process information (i.e. read sentences) under divided attention. Operation span is very similar to reading span except that the processing component is the performance of mathematical operations. While reading-span is by and large the only WM task used in language research, both tasks have been shown to predict sentence comprehension performance (Turner \& Engle, 1989). $N$-back performance reflects the ability to maintain, monitor and regularly update information. The relationship between $n$-back and sentence processing is unclear and largely untested. Sprouse, Wagers and Phillips (2012) found no evidence of a relationship between $n$-back performance and island effects (i.e. effects of syntactic complexity) on acceptability judgments. Novick and colleagues, however, found that individuals who improve their $n$-back performance via training show reduced garden-path effects in their comprehension accuracy relative to those who do not respond to training (Novick, Hussey, Teubner-Rhodes, Dougherty, Harbison \& Bunting, in press). The anti-saccade task tests the ability to suppress a prepotent, goal-irrelevant response. Bilinguals are known to out-perform monolinguals in tasks tapping this skill (Bialystok, Craik, \& Luk, 2008), suggesting a possible connection with language processing. By including a wider range of working memory assessments, the current study aimed to enrich understanding of the cognitive underpinnings of sentence processing. 


\section{Methods}

\section{Participants}

Data was collected from 44 right handed participants (25 female) between the ages of 18 and 40 (mean age $=21.9$, S.D. $=3.56$ ) All participants were neurologically normal, native speakers of English. None had had started learning a second language before age 12.

\section{Sentence Stimuli}

The sentence stimuli consisted of garden-path, object relative and control sentences (see 3-5 above, respectively, most of which came from Gouvea, Phillips \& Kazanina and Poeppel (2010)'s stimuli. Each list contained 36 sentences in each of the three conditions. The presentation of the 108 sentences was counterbalanced such that each sentence appeared in one condition per list. In addition, 72 filler sentences (matched for length and complexity) were included. Fifty percent of the non-filler sentences were followed by a comprehension question (25\% for fillers). Questions came either from Gouvea et al. (2010)'s stimuli or were created for the new sentences. In total, six lists were created such that each sentence appeared in each condition, with or without a comprehension question, across lists.

1. The patient met the doctor and the nurse with the white dress showed the chart during the meeting. (Garden-Path)

2. The patient met the doctor to whom the nurse with the white dress showed the chart during the meeting. (Object Relative)

3. The patient met the doctor while the nurse with the white dress showed the chart during the meeting. (Control)

\section{Working Memory Tasks}

Reading span Automated Reading-Span (Unsworth, Heitz, Schrock \& Engle, 2005) was used in this experiment. Participants were presented with a series of sentences and asked to indicate, via button press, if the sentences make sense. After each sentence they were then presented with a letter that they must remember. At the end of the sequence, they had to recall the letters in the order of presentation. Their score reflects the total number of letters recalled in the correct order.

Operation span Automated Operation-Span (Unsworth et al., 2005) was used in this experiment. Operation span is identical to reading span as described above except instead of making sense judgments on sentences, participants had to solve math problems involving multiple operations.

$N$-back In the $n$-back task, participants were presented with a sequence of single letters and asked to judge if the current letter is the same as the one that occurred $n$ places back in the sequence. For example, in a 4-back task, the third " $X$ " in the following sequence would be a target: X U P X X U U. Lures, which appeared one space before a target ( $n-1$; the second “X”) or one space after a target $(n+1$; the third " $U$ ”) were also included. Participants in the current experiment performed 2-back and 4-back. Accuracy for four item types (target, non-target, $n$ - 1 lure and $n+1$ lure) were averaged across $n$ level (2-back and 4-back). 
Anti-saccade In the anti-saccade task, participants performed a letter monitoring task. They were first presented with a flashing cue that appears on either the left or right side of the computer screen. The cue was followed by a letter. The letter was either on the same side of the screen as the cue (pro-saccade) or on the opposite side (anti-saccade). Participants had to suppress the impulse to shift their gaze to the cue in order to maximize performance.

\section{Procedure}

Participants performed the sentence processing task first. Sentences were presented word by word such that each word was on the screen for $300 \mathrm{~ms}$, followed by a $200 \mathrm{~ms}$ blank. Questions were presented in their entirety for $2500 \mathrm{~ms}$. There was a $3500 \mathrm{~ms}$ break between each item. After the sentence processing task, participants completed the four working memory assessments.

\section{Results}

Accuracy was lower for garden-path (77.5\%, S.D. 17.1) and object relative (76.9\%, S.D. 19.6) sentences than for controls $(83.3 \%, 13.9)$. There were significant differences between gardenpaths and controls $(F(1,43)=4.18, p<.05)$ and object relatives and controls $(F(1,43)=5.44, p<$ $.05)$. The correlational analysis of the working memory assessments showed significant correlations between operation span and reading span $(r=.428, p<.01)$, operation span and anti-saccade accuracy $(r=.308 . p<.05)$, and anti-saccade and $n$-back target accuracy $(r=.308$, $p=.05)$. There were significant correlations with operation span and comprehension accuracy for control ( $r=.345, p<.05)$, garden-path $(r=.362, p<.05)$ and object relative $(r=.454, p<$ .005) sentences. Reading span, on the other hand, only correlated with accuracy for control sentences $(r=.497, p<.005)$. Similarly, anti-saccade correlated with accuracy for control sentences $(r=.315, p<.05)$.

\section{Discussion}

Reading Span, Operation Span and Anti-saccade predict comprehension of simple sentences. Only Operation Span predicts accuracy for syntactically complex sentences. N-back accuracy does not correlate with comprehension performance. The finding for Operation Span confirms that the ability to store and process under divided attention underpins the interpretation of complex syntax. The Reading Span findings suggest that the processing component of the task (reading simple sentences) was not sufficiently demanding to predict individual differences in the interpretation of the syntactically complex sentences in this study. The finding for antisaccade indicates that the increased processing demands of complex syntax do not involve the resolution of conflict between potent response and task goal. The relationship between n-back and language processing remains unclear. These results provide evidence that sentence comprehension may be underpinned by distinct subcomponents of working memory and that the relationship between sentence comprehension and working memory mechanisms is modulated by syntactic complexity. 


\section{References}

Bialystok, E., Craik, F. I. M. \& Luk, G. (2008). Cognitive control and lexical access in younger and older bilinguals. Journal of Experimental Psychology: Learning, Memory and Cognition, 34, 859-873.

Caplan, D., \& Waters, G.S. (1999). Verbal working memory and sentence comprehension. Behavioral and Brain Sciences, 22, 77-126.

Chiappe, D.L. \& Chiappe, P. (2007). The role of working memory in metaphor production and comprehension. Journal of Memory and Language, 56(2), 172-188.

Conway, A.R., Kane, M.J., Bunting, M.F., Hambrick, D.Z., Wilhelm, O. \& Engle, R.W. (2005). Working memory span tasks: A methodological review and user's guide. Psychonomic Bulletin \& Review, 12(5), 769-786.

Daneman, M., \& Carpenter, P.A. (1980). Individual differences in working memory and reading. Journal of Verbal Learning and Verbal Behavior, 19, 450-466.

Ferreira, F., Bailey, K.G.D., Ferraro, V., 2002. Good-enough representations in language comprehension. Current Directions in Psychological Science, 11, 11-15.

Frazier, L. (1987). Syntactic processing: Evidence from Dutch. Natural Language and Linguistic Theory, 5, 519-559.

Frazier, L. \& Rayner, K. (1982). Making and correcting errors during sentence comprehension: eye movements in the analysis of structurally ambiguous sentences. Cognitive Psychology, $14,178-210$.

Friederici, A. D., Steinhauer, K., Mecklinger, A., \& Meyer, M. (1998). Working memory constraints on syntactic ambiguity resolution as revealed by electrical brain responses. Biological Psychology, 47, 193-221.

Gouvea, A.C., Phillips, C., Kazanina, N. \& Poeppel, D. (2010). The linguistic processes underlying the P600. Language and Cognitive Processes, 25(2), 149-188.

Novick, J. M., Hussey, E., Teubner-Rhodes, S., Dougherty, M. R., Harbison, J. I., \& Bunting, M. F. (in press). Clearing the garden-path: Improving sentence processing through executive control training. Language and Cognitive Processes.

Just, M.A., \& Carpenter, P.A. (1992). A capacity theory of comprehension: Individual differences in working memory. Psychological Review, 99, 122-149.

Sprouse, J., Wagers, M. \& Philips, C. (2012). A test of the relation between working-memory capacity and syntactic island effects. Language, 88(1), 82-123.

Turner, M.L. \& Engle, R.W. (1989). Is working memory capacity task independent? Journal of Memory and Language, 28, 127-154.

Unsworth, N., Schrock, J.C. \& Engle, R.W. (2004). Working memory capacity and the antisaccade task: Individual differences in voluntary saccade control. Journal of Experimental Psychology: Learning Memory and Cognition, 30, 1302-1321.

Vos, S.H., Gunter, T., Schriefers, H. y Friederici, A.D. (2001). Syntactic parsing and working memory: The effects of syntactic complexity, reading span and concurrent load. Language and Cognitive Processes, 16, 65-103. 